\title{
OPERATOR INTERPRETATION OF RESONANCES GENERATED BY SOME OPERATOR MATRICES*†
}

\author{
R. MENNICKEN ${ }^{\ddagger}$, A. K. MOTOVILOV ${ }^{\S}$
}

\begin{abstract}
We consider the analytic continuation of the transfer function for a $2 \times 2$ matrix Hamiltonian into the unphysical sheets of the energy Riemann surface. We construct a family of non-selfadjoint operators which reproduce certain parts of the transfer-function spectrum including resonances situated on the unphysical sheets neighboring the physical sheet. On this basis, completeness and basis properties for the root vectors of the transfer function (including those for the resonances) are proved.
\end{abstract}

\section{Introduction}

In this paper we deal with $2 \times 2$ operator matrices

$$
\mathbf{H}=\left(\begin{array}{cc}
A_{0} & B_{01} \\
B_{10} & A_{1}
\end{array}\right)
$$

acting in an orthogonal sum $\mathcal{H}=\mathcal{H}_{0} \oplus \mathcal{H}_{1}$ of separable Hilbert spaces $\mathcal{H}_{0}$ and $\mathcal{H}_{1}$. The entries $A_{0}: \mathcal{H}_{0} \rightarrow \mathcal{H}_{0}$, and $A_{1}: \mathcal{H}_{1} \rightarrow \mathcal{H}_{1}$, are assumed to be self-adjoint operators with domains $\mathcal{D}\left(A_{0}\right)$ and $\mathcal{D}\left(A_{1}\right)$, respectively. It is assumed that the couplings $B_{i j}: \mathcal{H}_{j} \rightarrow \mathcal{H}_{i}, i, j=0,1$, $i \neq j$, are bounded operators (i. e., $\left.B_{i j} \in \mathbf{B}\left(\mathcal{H}_{j}, \mathcal{H}_{i}\right)\right)$ and $B_{01}=B_{10}^{*}$. Under these assumptions the matrix $\mathbf{H}$ is a self-adjoint operator in $\mathcal{H}$ with domain $\mathcal{D}(\mathbf{H})=\mathcal{D}\left(A_{0}\right) \oplus \mathcal{D}\left(A_{1}\right)$. Note that operators of the form (1]) arise in many of quantum-physical problems (see e.g., [1] - [7]).

In the spectral theory of operator matrices (1) an important role is played by the transfer functions

$$
M_{i}(z)=A_{i}-z+V_{i}(z) \quad \text { where } \quad V_{i}(z)=-B_{i j} R_{j}(z) B_{j i}, \quad i=0,1, \quad j \neq i .
$$

${ }^{*}$ LANL E-print math.SP/9809093. Contribution to Proceedings of the Mark Krein International Conference on Operator Theory and Applications, Odessa, August 18-22, 1997.

${ }^{\dagger}$ Financial support of this work by the DFG, INTAS and RFBR is kindly acknowledged.

${ }^{\ddagger}$ Address: Naturwiss. Fakultät I - Mathematik, Universität Regensburg, D-93040 Regensburg, Germany. E-mail: reinhard.mennicken@mathematik.uni-regensburg.de.

$\S$ Address: Physikalishes Institut, Universität Bonn, Endenicher Allee 11-13, D-53115 Bonn, Germany. On leave of absence from the Bogoliubov Laboratory of Theoretical Physics, Joint Institute for Nuclear Research, 141980 Dubna, Russia. E-mail: motovilov@physik.uni-bonn.de and motovilv@thsun1.jinr.ru. 
A particular role of the functions $M_{i}(z)$ can be understood already from the fact that the resolvent of the operator (11) can be expressed explicitly in terms of the inverse transfer functions $M_{0}^{-1}(z)$ or $M_{1}^{-1}(z)$. Therefore, in studying the spectral properties of the transfer functions one studies at the same time the spectral properties of the operator matrix $\mathbf{H}$.

In the papers [8], [9] the following question was raised: Is it possible to introduce an operator $H_{i}, i=0,1$, independent of the spectral parameter $z$, such that the equality $H_{i} \psi^{(i)}=z \psi^{(i)}, \psi^{(i)} \in \mathcal{H}_{i}$, implies $M_{i}(z) \psi^{(i)}=0$ ? Obviously, having found such an operator one would reduce the spectral problem for the transfer-function $M_{i}(z)$ to the standard spectral problem for the operator $H_{i}$ and, thus, the completeness and basis properties for the eigenvectors of $M_{i}$ could be studied in terms of the operator $H_{i}$ referring to well known facts from operator theory. A rigorous answer to the above question was found in [10], [11] in the case where the spectra $\sigma\left(A_{0}\right)$ and $\sigma\left(A_{1}\right)$ of the entries $A_{0}$ and $A_{1}$ are separated from each other,

$$
\operatorname{dist}\left\{\sigma\left(A_{0}\right), \sigma\left(A_{1}\right)\right\}>0 .
$$

To this end an operator-valued function $V_{i}\left(Y_{i}\right)$ on the space of linear operators in $\mathcal{H}_{i}$ was constructed in [10], [11] such that $V_{i}\left(Y_{i}\right) \psi^{(i)}=V_{i}(z) \psi^{(i)}$ for any eigenvector $\psi^{(i)}$ corresponding to an eigenvalue $z$ of the operator $Y_{i}$. The desired operator $H_{i}$ was searched for as a solution of the operator equation

$$
H_{i}=A_{i}+V_{i}\left(H_{i}\right), \quad i=0,1 .
$$

Notice that an equation of the form (4) first appeared explicitly in the paper [12 by M. A. Braun.

The solvability of the equation (4) was announced in [10] and proved in [11] under the assumption $\left\|B_{i j}\right\|_{2}<\frac{1}{2} \operatorname{dist}\left\{\sigma\left(A_{0}\right), \sigma\left(A_{1}\right)\right\}$ where $\left\|B_{i j}\right\|_{2}$ stands for the Hilbert-Schmidt norm of the couplings $B_{i j}$. It was found in [11] that the problem of constructing the operators $H_{i}$ is closely related to the problem of searching for the invariant subspaces of the matrix $\mathbf{H}$ which admit representation in the form of graphs for some bounded $Q_{j i}: \mathcal{H}_{i} \rightarrow \mathcal{H}_{j}$. The point is that under the conditions of [10], [11] the solutions $H_{i}, i=0,1$, of Eqs. ([4) read $H_{i}=A_{i}+B_{i j} Q_{j i}$ while $Q_{j i}$ are contractions. The operators $Q_{j i}$ determine a similarity transform reducing the matrix $\mathbf{H}$ to the block-diagonal form $\mathbf{H}^{\prime}=\operatorname{diag}\left\{H_{0}, H_{1}\right\}$ (see [11]).

The idea of the block diagonalization of the $2 \times 2$ operator matrices in terms of the invariant subspaces allowing a graph representation goes back yet to the paper [3] by S. OKubo (regarding applications of Okubo's approach to particle physics see, e. g., Refs. [3] - [5]). In a mathematically rigorous way this idea was applied by V. A. MALYSHEV and R. A. Minlos [6] to a class of selfadjoint operators in statistical physics. The techniques of Ref. [6] are restricted to the case where the norms of the entries $B_{i j}$ are sufficiently small and the separation condition (3) holds, too. V. M. AdAmyan and H. LANGer [13] proved the existence of invariant subspaces allowing a graph representation for arbitrary bounded entries $B_{i j}$ however assuming, instead of the condition (3), the essentially different assumption that the spectrum of one of the entries $A_{i}, i=0,1$ is situated strictly below the spectrum of the other one, say $\max \sigma\left(A_{1}\right)<\min \sigma\left(A_{0}\right)$. Recently, the result of 113 was extended by V. M. Adamyan, H. Langer, R. Mennicken and J. Saurer [14 to the case where $\max \sigma\left(A_{1}\right) \leq \min \sigma\left(A_{0}\right)$ and where the couplings $B_{i j}$ were allowed to be unbounded operators such that, for $\alpha_{0}<\min \sigma\left(A_{0}\right)$, the product $\left(A_{0}-\alpha_{0}\right)^{-1 / 2} B_{01}$ makes 
sense as a bounded operator. The mentioned conditions were then somewhat weakened by R. Mennicken and A. A. Shkalikov [15] in the case of a bounded entry $A_{1}$ and the same type of entries $B_{i j}$ as in [14]. Instead of the explicit conditions on the spectra of $A_{i}$, the paper [15] uses a condition on the spectrum of the transfer function $M_{1}(z)$ itself. One can check that the spectral component $H_{i}$ of the matrix $\mathbf{H}$ constructed in [13 - 15] satisfies the equation (4), at least in the case where for $j \neq i$ the entry $A_{j}$ is bounded.

In the present work we study the equation (雨) in a case which is totally different from the spectral situations considered in [6], [10], [11], [13]-[15]. From the beginning, we suppose that $\sigma\left(A_{0}\right) \cap \sigma\left(A_{1}\right) \neq \emptyset$. Moreover, we are especially interested just in the case where the spectrum of, say $A_{1}$, is partly or totally embedded into the continuous spectrum of $A_{0}$. We work under the assumption that the coupling operators $B_{i j}$ are such that the transfer function $M_{1}(z)$ admits analytic continuation, as an operator-valued function, under the cuts along the branches of the absolutely continuous spectrum $\sigma_{a c}\left(A_{0}\right)$ of the entry $A_{0}$. In Sect. 2 we describe the conditions on $B_{i j}$ making such a continuation of $M_{1}(z)$ possible.

The problem considered is closely related to the resonances generated by the matrix H. Regarding a definition of the resonance and history of the subject see, e. g., the book [16]. A recent survey of the literature on resonances can be found in [17]. Throughout the paper we treat resonances as the discrete spectrum of the transfer function $M_{1}(z)$ situated in the unphysical sheets of its Riemann surface. We assume that the absolutely continuous spectrum of the entry $A_{0}$ consists of $m(1 \leq m<\infty)$ distinct intervals. As a result in Sect. 3 we get $2^{m}$ variants of the function $V_{1}(Y)$ and, consequently, $2^{m}$ different variants of the equation (4) which read now as Eq. (12). The solutions of (12) represent non-selfadjoint operators the spectrum of which includes the resonances in unphysical sheets neighboring the physical one.

In Sect. 1 we first prove the factorization theorem for the transfer function $M_{1}(z)$. It follows from this theorem that there exist certain domains surrounding the set $\sigma\left(A_{1}\right)$ and lying partly in the unphysical sheet(s) where the spectrum of $M_{1}$ is represented only by the spectrum of the respective solutions of the basic equation (12). Since the root vectors of these solutions are also root vectors for $M_{1}$, this fact allows us to talk further, in Sect. 5 and 6, about completeness and basis properties of the root vectors of the transfer function $M_{1}$ corresponding to its spectrum in the above domains, including the resonance spectrum. To prove these properties we rely mainly on the respective statements from the books by I. C. Gohberg and M. G. Krein [18] and by T. Kato [19].

\section{Analytic continuation of the transfer function}

The transfer function $M_{i}(z), i=0,1$, considered on the resolvent set $\rho\left(A_{j}\right)$ of the entry $A_{j}$, $j \neq i$, represents a particular case of a holomorphic operator-valued function. In the present work we use the standard definition of holomorphy of an operator-valued function with respect to the operator norm topology (see, e.g., [14]). One can extend to operator-valued functions the usual definitions of the spectrum and its components. Each transfer function $M_{i}(z), i=0,1$, is holomorphic at least in the resolvent set $\rho\left(A_{j}\right)$ of the entry $A_{j}, j \neq i$. Since the inverse transfer functions $M_{i}^{-1}(z)$ coincide with the respective block components 
$\mathbf{R}_{i i}(z)$ of the resolvent $\mathbf{R}(z)=(\mathbf{H}-z)^{-1}$, they are both holomorphic at least in the set $\rho(\mathbf{H})$. Let $E_{j}$ be the spectral measure for the entry $A_{j}, A_{j}=\int_{\sigma\left(A_{j}\right)} \lambda d E_{j}(\lambda), j=0,1$, $\sigma\left(A_{j}\right) \subset \mathbb{R}$. Then the functions $V_{i}(z)$ can be written

$$
V_{i}(z)=B_{i j} \int_{\sigma\left(A_{j}\right)} d E_{j}(\mu) \frac{1}{z-\mu} B_{j i} .
$$

Thus, it is convenient to introduce the quantities $\mathcal{V}_{j}(B)=\sup _{\left\{\delta_{k}\right\}} \sum_{k}\left\|B_{i j} E_{j}\left(\delta_{k}\right) B_{j i}\right\|$, with $\left\{\delta_{k}\right\}$ being a finite or countable complete system of Borel subsets of $\sigma\left(A_{j}\right)$ such that $\delta_{k} \cap \delta_{l}=$ $\emptyset$, if $k \neq l$ and $\bigcup_{k} \delta_{k}=\sigma\left(A_{j}\right)$. The number $\mathcal{V}_{j}(B)$ is called the variation of the operators $B_{i j}$ with respect to the spectral measure $E_{j}$. Along with the "total" variation $\mathcal{V}_{j}(B)$ we shall use the "truncated" variations $\left.\mathcal{V}_{j}(B)\right|_{\Delta}=\sup _{\left\{\delta_{k}\right\}} \sum_{k}\left\|B_{i j} E_{j}\left(\delta_{k} \cap \Delta\right) B_{j i}\right\|$ where $\Delta$ is a certain Borel subset of $\sigma\left(A_{j}\right) ;\left.\mathcal{V}_{j}(B)\right|_{\Delta} \leq \mathcal{V}_{j}(B)$.

The value of $\mathcal{V}_{j}(B)$ satisfies the estimates $\left\|B_{i j}\right\|^{2} \leq \mathcal{V}_{j}(B) \leq\left\|B_{i j}\right\|_{2}^{2}$. The equality $\mathcal{V}_{j}(B)=\left\|B_{i j}\right\|^{2}$ is attained in the case where $A_{j}$ is a multiple of the identity operator. The equality $\mathcal{V}_{j}(B)=\left\|B_{i j}\right\|_{2}^{2}$ holds if $A_{j}$ possesses only a pure discrete spectrum which is at the same time simple.

We assume that the spectrum of the operator $A_{1}$ can intersect only the continuous spectrum of the operator $A_{0}$ and this intersection is only realized on (every of) the pairwise nonintersecting open intervals $\Delta_{k}^{0}=\left(\mu_{k}^{(1)}, \mu_{k}^{(2)}\right) \subset \sigma_{c}\left(A_{0}\right), \mu_{k}^{(1)}<\mu_{k}^{(2)}, k=1,2, \ldots, m$, $m<\infty$, and $-\infty \leq \mu_{1}^{(1)}, \mu_{m}^{(2)} \leq+\infty$. Therefore, we assume that $\Delta_{k}^{0} \cap \sigma\left(A_{1}\right) \neq \emptyset$ for all $k=1,2, \ldots, m$ and $\sigma\left(A_{1}\right) \cap \sigma^{\prime}\left(A_{0}\right)=\emptyset$ where $\sigma^{\prime}\left(A_{0}\right)=\sigma\left(A_{0}\right) \backslash \bigcup_{k=1}^{m} \Delta_{k}^{0}$.

We shall suppose that the product $K_{B}(\mu) \stackrel{\text { def }}{=} B_{10} E^{0}(\mu) B_{01}$ where $E^{0}(\mu)$ stands for the spectral function of $A_{0}, E^{0}(\mu)=E_{0}((-\infty, \mu))$, is differentiable in $\mu \in \Delta_{k}^{0}, k=$ $1,2, \ldots, m$, in the operator norm topology. The derivative $K_{B}^{\prime}(\mu)$ is non-negative, $K_{B}^{\prime}(\mu) \geq$ 0 , since $K_{B}(\mu)$ is a non-decreasing function. Differentiability of $K_{B}(\mu)$ means that the continuous spectrum of the entry $A_{0}$ includes, in each $\Delta_{k}^{0}, k=1,2, \ldots, m$, a branch of the absolutely continuous spectrum $\sigma_{a c}\left(A_{0}\right)$. Obviously, $\left.\mathcal{V}_{0}(B)\right|_{\Delta_{k}^{0}}=\int_{\Delta_{k}^{0}} d \mu\left\|K_{B}^{\prime}(\mu)\right\|$.

Further, we suppose that the function $K_{B}^{\prime}(\mu)$ is continuous within the closed intervals $\overline{\Delta_{k}^{0}}$ and, moreover, that it admits analytic continuation from each of these intervals to a simply connected domain situated, say, in $\mathbb{C}^{-}$. For the interval $\Delta_{k}^{0}$, let this domain be called $D_{k}^{-}$. We assume that the boundary of each domain $D_{k}^{-}$includes the entire spectral interval $\Delta_{k}^{0}$ and the domains $D_{k}^{-}$and $D_{j}^{-}$for different $k$ and $j$ do not intersect each other. Since $K_{B}^{\prime}(\mu)$ represents a self-adjoint operator for $\mu \in \Delta_{k}^{0}$ and $\Delta_{k}^{0} \subset \mathbb{R}$, the function $K_{B}^{\prime}(\mu)$ also automatically admits analytic continuation from $\Delta_{k}^{0}$ into the domain $D_{k}^{+}$, symmetric to $D_{k}^{-}$ with respect to the real axis, $D_{k}^{+}=\left\{z: \bar{z} \in D_{k}^{-}\right\}$. For the continuation into $D_{k}^{+}$we retain the same notation $K_{B}^{\prime}(\mu)$. The selfadjoitness of $K_{B}^{\prime}(\mu)$ for $\mu \in \Delta_{k}^{0}$ implies $\left[K_{B}^{\prime}(\mu)\right]^{*}=K_{B}^{\prime}(\bar{\mu})$, $\mu \in D_{k}^{ \pm}$. Also, we shall always suppose the $K_{B}^{\prime}(\mu)$ satisfies the Hölder condition at the (finite) end points $\mu_{k}^{(1)}, \mu_{k}^{(2)}$ of the spectral intervals $\Delta_{k}^{0},\left\|K_{B}^{\prime}(\mu)-K_{B}^{\prime}\left(\mu_{k}^{(i)}\right)\right\| \leq C\left|\mu-\mu_{k}^{(i)}\right|^{\gamma}$, $i=1,2, \mu \in D_{k}^{ \pm}$, with some positive $C$ and $\gamma$.

Let $l=\left(l_{1}, l_{2}, \ldots, l_{m}\right)$ be a multi-index having the components $l_{k}=+1$ or $l_{k}=-1$, $k=1,2, \ldots, m$. In what follows we consider the domains $D_{l}=\bigcup_{k=1}^{m} D_{k}^{l_{k}}$. Let $\Gamma_{k}^{l_{k}}$ be a 
rectifiable Jordan curve in $D_{k}^{l_{k}}$ resulting from continuous deformation of the interval $\Delta_{k}^{0}$, the (finite) end points of this interval being fixed. With the exception of the end points, the closure $\bar{\Gamma}_{k}^{l_{k}}$ of the contour $\Gamma_{k}^{l_{k}}$ should have no other common points with the set $\sigma_{c}\left(A_{0}\right)$. By $\Gamma_{l}, l=\left(l_{1}, l_{2}, \ldots, l_{m}\right)$, we shall denote the union of the contours $\Gamma_{k}^{l_{k}}, \Gamma_{l}=\bigcup_{k=1}^{m} \Gamma_{k}^{l_{k}}$. Also, we extend the definition of the variation $\mathcal{V}_{0}(B)$ to the set $\sigma^{\prime}\left(A_{0}\right) \cup \Gamma_{l}$ by introducing the modified variation

$$
\mathcal{V}_{0}\left(B, \Gamma_{l}\right)=\left.\mathcal{V}_{0}(B)\right|_{\sigma^{\prime}\left(A_{0}\right)}+\int_{\Gamma_{l}}|d \mu|\left\|K_{B}^{\prime}(\mu)\right\|
$$

with $|d \mu|$ Lebesgue measure on $\Gamma_{l}$. We suppose that the operators $B_{i j}$ are such that there exists a contour (contours) $\Gamma_{l}$ where the value $\mathcal{V}_{0}\left(B, \Gamma_{l}\right)$ is finite, $\mathcal{V}_{0}\left(B, \Gamma_{l}\right)<\infty$, including also the case of the unbounded set $\bigcup_{k=1}^{m} \Delta_{k}^{0}$. The contours $\Gamma_{l}$ satisfying the condition $\mathcal{V}_{0}\left(B, \Gamma_{l}\right)<\infty$ are said to be $K_{B}$-bounded contours.

LEMMA 1 The analytic continuation of the transfer function $M_{1}(z), z \in \mathbb{C} \backslash \sigma\left(A_{0}\right)$, through the spectral intervals $\Delta_{k}^{0}$ into the subdomain $D\left(\Gamma_{l}\right) \subset D_{l}$ bounded by the set $\bigcup_{k=1}^{m} \Delta_{k}^{0}$ and a $K_{B}$-bounded contour $\Gamma_{l}$ is given by

$$
M_{1}\left(z, \Gamma_{l}\right)=A_{1}-z+V_{1}\left(z, \Gamma_{l}\right)
$$

where

$$
\begin{aligned}
V_{1}\left(z, \Gamma_{l}\right) & =\int_{\sigma^{\prime}\left(A_{0}\right) \cup \Gamma_{l}} K_{B}(d \mu) \frac{1}{z-\mu} \\
& \stackrel{\text { def }}{=} \int_{\sigma^{\prime}\left(A_{0}\right)} B_{10} E_{0}(d \mu) B_{01} \frac{1}{z-\mu}+\int_{\Gamma_{l}} d \mu K_{B}^{\prime}(\mu) \frac{1}{z-\mu}
\end{aligned}
$$

For $z \in D_{k}^{l_{k}} \cap D\left(\Gamma_{l}\right)$ the function $M_{1}\left(z, \Gamma_{l}\right)$ may be written as

$$
M_{1}\left(z, \Gamma_{l}\right)=M_{1}(z)+2 \pi \mathrm{i} l_{k} K_{B}^{\prime}(z)
$$

$\mathrm{Pr}$ o o f. The proof is reduced to the observation that the function $M_{1}\left(z, \Gamma_{l}\right)$ is holomorphic for $z \in \mathbb{C} \backslash\left[\sigma^{\prime}\left(A_{0}\right) \cup \Gamma_{l}\right]$ and coincides with $M_{1}(z)$ for $z \in \mathbb{C} \backslash\left[\sigma^{\prime}\left(A_{0}\right) \cup \overline{D\left(\Gamma_{l}\right)}\right]$. Eq. (8) is obtained from (17) using the Residue Theorem.

The formula (8) shows that in general the transfer function $M_{1}$ has a multi-sheeted Riemann surface. The sheet of the complex plane where the transfer function $M_{1}(z)$ is considered together with the resolvent $\mathbf{R}(z)$ initially is said to be the physical sheet. The remaining sheets of the Riemann surface of $M_{1}(z)$ are said to be unphysical sheets. In the present work we only deal with the unphysical sheets neighboring the physical one, i. e., with the sheets connected through the intervals $\Delta_{k}^{0}$ for some $k \in\{1,2, \ldots, m\}$ immediately to the physical sheet.

\section{The basic equation and its solutions}

If an operator-valued function $T: \sigma^{\prime}\left(A_{0}\right) \cup \Gamma \rightarrow \mathbf{B}\left(\mathcal{H}_{1}, \mathcal{H}_{1}\right)$ is continuous and bounded

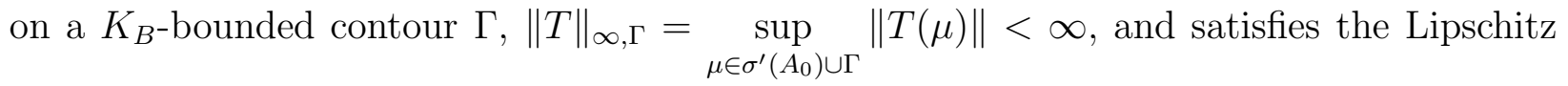


condition on $\sigma^{\prime}\left(A_{0}\right)$ then the integral

$$
\int_{\sigma^{\prime}\left(A_{0}\right) \cup \Gamma} K_{B}(d \mu) T(\mu) \stackrel{\text { def }}{=} \int_{\sigma^{\prime}\left(A_{0}\right)} B_{10} E_{0}(d \mu) B_{01} T(\mu)+\int_{\Gamma} d \mu K_{B}^{\prime}(\mu) T(\mu)
$$

exists in the sense of the operator norm topology (see Lemma 7.2 in [14]) and

$$
\left\|\int_{\sigma^{\prime}\left(A_{0}\right) \cup \Gamma} K_{B}(d \mu) T(\mu)\right\| \leq \mathcal{V}_{0}(B, \Gamma)\|T\|_{\infty, \Gamma} .
$$

In particular, if $T(z)$ is the resolvent of an operator $Y, T(z)=\left(Y-z I_{1}\right)^{-1}$, the spectrum of which is separated from $\sigma^{\prime}\left(A_{0}\right) \cup \Gamma$, then one can define the operator

$$
V_{1}(Y, \Gamma)=\int_{\sigma^{\prime}\left(A_{0}\right) \cup \Gamma} K_{B}(d \mu)(Y-\mu)^{-1}
$$

This operator is bounded, $V_{1}(Y, \Gamma) \in \mathbf{B}\left(\mathcal{H}_{1}, \mathcal{H}_{1}\right)$, and, because of (10), its norm admits the estimate

$$
\left\|V_{1}(Y, \Gamma)\right\| \leq \mathcal{V}_{0}\left(B, \Gamma_{l}\right) \sup _{\mu \in \sigma^{\prime}\left(A_{0}\right) \cup \Gamma}\left\|(Y-\mu)^{-1}\right\|
$$

In what follows we consider the equation

$$
Y=A_{1}+V_{1}(Y, \Gamma) .
$$

This equation possesses the following characteristic property: If an operator $H_{1}$ is a solution of (12) and $u_{1}$ is an eigenvector of $H_{1}, H_{1} u_{1}=z u_{1}$, then automatically $z u_{1}=A_{1} u_{1}+$ $V_{1}\left(H_{1}, \Gamma\right) u_{1}=A_{1} u_{1}+V_{1}(z, \Gamma) u_{1}$. This implies that any eigenvalue $z$ of such an operator $H_{1}$ is automatically an eigenvalue for the continued transfer function $M_{1}\left(z, \Gamma_{l}\right)$ and $u_{1}$ is its eigenvector. Thus, having found the solution(s) of the equation (12) one obtains an effective means of studying the spectral properties of the transfer function $M_{1}(z, \Gamma)$ itself. Often it turns out to be convenient to rewrite Eq. (12) in the form

$$
X=V_{1}\left(A_{1}+X, \Gamma\right)
$$

where $X=Y-A_{1}$.

THEOREM 1 Let a contour $\Gamma$ be $K_{B}$-bounded and

$$
\mathcal{V}_{0}(B, \Gamma)<\frac{1}{4} d_{0}^{2}(\Gamma)
$$

where $d_{0}(\Gamma)=\operatorname{dist}\left\{\sigma\left(A_{1}\right), \sigma^{\prime}\left(A_{0}\right) \cup \Gamma\right\}$. Then Eq. (13) is uniquely solvable in any ball $\mathcal{S}_{1}(r) \subset$ $\mathbf{B}\left(\mathcal{H}_{1}, \mathcal{H}_{1}\right)$ including operators $X: \mathcal{H}_{1} \rightarrow \mathcal{H}_{1}$ the norms of which are bounded as $\|X\| \leq r$ with $r$ such that

$$
r_{\min }(\Gamma) \leq r<r_{\max }(\Gamma)
$$

Here

$$
r_{\min }(\Gamma)=d_{0}(\Gamma) / 2-\sqrt{d_{0}^{2}(\Gamma) / 4-\mathcal{V}_{0}(B, \Gamma)}, \quad r_{\max }(\Gamma)=d_{0}(\Gamma)-\sqrt{\mathcal{V}_{0}(B, \Gamma)} .
$$

The solution $X$ of Eq. (13) is the same for any $r$ satisfying (15) and in fact it belongs to the smallest ball $\mathcal{S}_{1}\left(r_{\min }\right),\|X\| \leq r_{\min }(\Gamma)$. 
P r o o f . Let $F(X)=V_{1}\left(A_{1}+X, \Gamma\right)$ with $X \in \mathcal{S}_{1}(r)$. To begin with we search for a condition under which the function $F$ is a contracting mapping of the ball $\mathcal{S}_{1}(r)$ into itself. Since, in view of (15) and (16) the condition $0<r<d_{0}, d_{0}=d_{0}(\Gamma)$ automatically holds, the spectrum of the operator $A_{1}+X$ does not intersect the set $\sigma^{\prime}\left(A_{0}\right) \cup \Gamma$. This means that for all $\mu \in \sigma^{\prime}\left(A_{0}\right) \cup \Gamma$ the resolvent $\left(A_{1}+X-\mu I_{1}\right)^{-1}$ exists as a bounded operator in $\mathcal{H}_{1}$. It follows from the estimate (11) that

$$
\|F(X)\| \leq \mathcal{V}_{0}(B, \Gamma) \sup _{\mu \in \sigma^{\prime}\left(A_{0}\right) \cup \Gamma}\left\|\left(A_{1}+X-\mu\right)^{-1}\right\| \leq \mathcal{V}_{0}(B, \Gamma) \frac{1}{d_{0}-r}
$$

while

$$
\|F(X)-F(Y)\| \leq \mathcal{V}_{0}(B, \Gamma) \frac{1}{\left(d_{0}-r\right)^{2}}\|Y-X\|
$$

It immediately follows from these that the ball $\mathcal{S}_{1}(r)$ is necessarily mapped by the function $F$ into itself and this mapping is a contraction if the radius $r$ and the value $\mathcal{V}_{0}(B, \Gamma)$ are such that

$$
\mathcal{V}_{0}(B, \Gamma) \leq r\left(d_{0}-r\right), \quad \mathcal{V}_{0}(B, \Gamma)<\left(d_{0}-r\right)^{2} .
$$

Under the condition (14) the inequalities (17) considered together are just equivalent to the condition (15). Thus if this condition is valid then Eq. (13) has a solution in any ball $\mathcal{S}_{1}(r)$ with $r$ satisfying (15) and this solution is unique. This means that the solution is the same for all the radii satisfying (15). Moreover, it belongs to the ball $\mathcal{S}_{1}\left(r_{\min }\right)$ with the radius $r_{\min }$ given by (16).

THEOREM 2 Let the conditions of Theorem 1 be valid for a $K_{B}$-bounded contour $\Gamma \subset D_{l}$ and let $X$ be the solution of Eq. (13) referred to there. Then $X$ coincides with the analogous solution $\tilde{X}$ for any other $K_{B}$-bounded contour $\tilde{\Gamma} \subset D_{l}$ satisfying the estimate $\mathcal{V}_{0}(B, \tilde{\Gamma})<$ $\tilde{d}_{0}^{2} / 4$ with $0<\tilde{d}_{0}=\operatorname{dist}\left\{\sigma\left(A_{1}\right), \sigma^{\prime}\left(A_{0}\right) \cup \tilde{\Gamma}\right\} \leq d_{0}(\Gamma)$. Moreover, this solution satisfies the inequality $\|X\| \leq r_{0}(B)$ wherem $r_{0}(B)=\inf _{\Gamma_{l}: \omega\left(B, \Gamma_{l}\right)>0} r_{\min }\left(\Gamma_{l}\right)$ with $r_{\min }\left(\Gamma_{l}\right)$ given by (16) and $\omega\left(B, \Gamma_{l}\right)=d_{0}^{2}\left(\Gamma_{l}\right)-4 \mathcal{V}_{0}\left(B, \Gamma_{l}\right)$. The value of $r_{0}(B)$ does not depend on $l$.

$\mathrm{P} \mathrm{r} \mathrm{o} \mathrm{o} \mathrm{f}$ of this theorem is reduced to an appropriate continuous deformation of the integration paths. An only essential point is a checking of independence of the radius $r_{0}(B)$ of the multi-index $l$. To be sure in such an independence we consider an arbitrary $K_{B}$-bounded contour $\Gamma_{l} \subset D_{l}, \Gamma_{l}=\bigcup_{k=1}^{m} \Gamma_{k}^{l_{k}}$. Denote by $\Gamma_{l^{\prime}}$ a contour resulting from $\Gamma_{l}$ after replacing a part of $\Gamma_{k}^{l_{k}}$ with curves $\Gamma_{k}^{\left(-l_{k}\right)}=\left\{\mu: \bar{\mu} \in \Gamma_{k}^{l_{k}}\right\}$, symmetric to $\Gamma_{k}^{l_{k}}$ with respect to the real axis. Obviously, such replacements generate, additionally to $\Gamma_{l}, 2^{m}-1$ different contours $\Gamma_{l^{\prime}}$ where $l^{\prime}=\left(l_{1}^{\prime}, l_{2}^{\prime}, \ldots, l_{m}^{\prime}\right)$ with $l_{k}^{\prime}= \pm l_{k}, k=1,2, \ldots, m$. For every of such contours the value of $\mathcal{V}_{0}\left(B, \Gamma_{l^{\prime}}\right)$ is the same, namely $\mathcal{V}_{0}\left(B, \Gamma_{l^{\prime}}\right)=\mathcal{V}_{0}\left(B, \Gamma_{l}\right)$, since the replacement of $\Gamma_{l}$ with $\Gamma_{l^{\prime}}$ does not change $\int_{\Gamma_{l}}|d \mu|\left\|K_{B}^{\prime}(\mu)\right\|$. But this just means that $r_{0}(B)$ does not depend on $l$.

So, for a given holomorphy domain $D_{l}$ the solutions $X$ and $H_{1}, H_{1}=A_{1}+X$, do not depend on the $K_{B}$-bounded contours $\Gamma_{l} \subset D_{l}$ satisfying the condition (14). But when 
the index $l$ changes, $X$ and $H_{1}$ can also change. For this reason we shall supply them in the following, when it is necessary, with the index $l$ writing, respectively, $X^{(l)}$ and $H_{1}^{(l)}$, $H_{1}^{(l)}=A_{1}+X^{(l)}$. Therefore, Theorem 1 guarantees us, in general, the existence of the $2^{m}$ solutions $X^{(l)}$ to the basic equation (12) and, hence, the $2^{m}$ respective solutions $H_{1}^{(l)}$ to the basic equation (13). Surely, Eqs. (12) and (13) are non-linear equations and, outside the balls $\|X\|<r_{\max }(\Gamma)$, they may, in principle, have other solutions, different from the $X^{(l)}$ or $H_{1}^{(l)}$ the existence of which is guaranteed by Theorem 1 . In the following we shall deal only with the solutions $X^{(l)}$ or $H_{1}^{(l)}$.

\section{Factorization of the transfer function}

THEOREM 3 Let $\Gamma_{l}$ be a $K_{B}$-bounded contour satisfying the condition (14) and $H_{1}^{(l)}=A_{1}+$ $X^{(l)}$ with $X^{(l)}$ the above solution of the basic equation (12), $\left\|X^{(l)}\right\| \leq r_{0}(B)$. Then, for $z \in \mathbb{C} \backslash\left(\sigma^{\prime}\left(A_{0}\right) \cup \Gamma_{l}\right)$, the transfer function $M_{1}\left(z, \Gamma_{l}\right)$ admits the factorization

$$
M_{1}\left(z, \Gamma_{l}\right)=W_{1}\left(z, \Gamma_{l}\right)\left(H_{1}^{(l)}-z\right)
$$

where $W_{1}\left(z, \Gamma_{l}\right)$ is a bounded operator in $\mathcal{H}_{1}$,

$$
W_{1}\left(z, \Gamma_{l}\right)=I_{1}-\int_{\sigma^{\prime}\left(A_{0}\right) \cup \Gamma_{l}} K_{B}(d \mu) \frac{1}{\mu-z}\left(H_{1}^{(l)}-\mu\right)^{-1} .
$$

Here, $I_{1}$ stands for the identity operator in $\mathcal{H}_{1}$.

For $\operatorname{dist}\left\{z, \sigma\left(A_{1}\right)\right\} \leq d_{0}\left(\Gamma_{l}\right) / 2$ the operator $W_{1}\left(z, \Gamma_{l}\right)$ is boundedly invertible and

$$
\left\|\left[W_{1}\left(z, \Gamma_{l}\right)\right]^{-1}\right\| \leq\left(1-\frac{\mathcal{V}_{0}\left(B, \Gamma_{l}\right)}{d_{0}^{2}\left(\Gamma_{l}\right) / 4}\right)^{-1}<\infty .
$$

Note that the above statement recalls the known factorization theorem by A. I. VIROZUB and V. I. MATSAEV [20] being valid for a class of selfadjoint operator-valued functions (see also [21]). However, in the case we deal with in the present paper, the function $M_{1}\left(z, \Gamma_{l}\right)$ it is not even a selfadjoint operator-valued function in the sense of [20].

P r o o f . For $z \in \mathbb{C} \backslash\left(\sigma^{\prime}\left(A_{0}\right) \cup \Gamma_{l}\right)$, the boundeness of the operator $W_{1}\left(z, \Gamma_{l}\right)$ given by (19) is evident. To prove the factorization (18) we note that for any $z \notin \sigma^{\prime}\left(A_{0}\right) \cup \Gamma_{l}$

$$
W_{1}\left(z, \Gamma_{l}\right)\left(H_{1}^{(l)}-z\right)=H_{1}^{(l)}-z-\int_{\sigma^{\prime}\left(A_{0}\right) \cup \Gamma_{l}} K_{B}(d \mu) \frac{\left(H_{1}^{(l)}-\mu\right)^{-1}\left(H_{1}^{(l)}-z\right)}{\mu-z} .
$$

Since $\left(H_{1}^{(l)}-\mu\right)^{-1}\left(H_{1}^{(l)}-z\right)=I_{1}+(\mu-z)\left(H_{1}^{(l)}-\mu\right)^{-1}$ one finds

$\int_{\sigma^{\prime}\left(A_{0}\right) \cup \Gamma_{l}} K_{B}(d \mu) \frac{\left(H_{1}^{(l)}-\mu\right)^{-1}\left(H_{1}^{(l)}-z\right)}{\mu-z}=\int_{\sigma^{\prime}\left(A_{0}\right) \cup \Gamma_{l}} \frac{K_{B}(d \mu)}{\mu-z}+\int_{\sigma^{\prime}\left(A_{0}\right) \cup \Gamma_{l}} K_{B}(d \mu)\left(H_{1}^{(l)}-\mu\right)^{-1}$.

But according to (6) $\int_{\sigma^{\prime}\left(A_{0}\right) \cup \Gamma_{l}} K_{B}(d \mu)(\mu-z)^{-1}=A_{1}-z-M_{1}\left(z, \Gamma_{l}\right)$, while according to (12) $\int_{\sigma^{\prime}\left(A_{0}\right) \cup \Gamma_{l}} K_{B}(d \mu)\left(H_{1}^{(l)}-\mu\right)^{-1}=H_{1}^{(l)}-A_{1}$. Making use of these expressions one immediately obtains Eq. (18). 
Further, we prove that the factor $W_{1}\left(z, \Gamma_{l}\right)$ is a boundedly invertible operator if the condition $\operatorname{dist}\left\{z, \sigma\left(A_{1}\right)\right\} \leq d_{0}\left(\Gamma_{l}\right) / 2$ is valid. Indeed, under this condition $|\mu-z| \geq$ $\operatorname{dist}\left\{z, \sigma^{\prime}\left(A_{0}\right) \cup \Gamma_{l}\right\} \geq d_{0}\left(\Gamma_{l}\right) / 2$ since $\operatorname{dist}\left\{\sigma\left(A_{1}\right), \sigma^{\prime}\left(A_{0}\right) \cup \Gamma_{l}\right\}=d_{0}\left(\Gamma_{l}\right)$. On the other hand $H_{1}^{(l)}=A_{1}+X^{(l)}$ and $\left\|X^{(l)}\right\|<d_{0}\left(\Gamma_{l}\right) / 2$. Thus for $\mu \in \sigma^{\prime}\left(A_{0}\right) \cup \Gamma_{l}$ we have $\left\|\left(H_{1}^{(l)}-\mu\right)^{-1}\right\|<$ $2 / d_{0}\left(\Gamma_{l}\right)$. Consequently

$$
\left\|\int_{\sigma^{\prime}\left(A_{0}\right) \cup \Gamma_{l}} K_{B}(d \mu) \frac{1}{\mu-z}\left(H_{1}^{(l)}-\mu\right)^{-1}\right\|<\frac{\mathcal{V}_{0}\left(B, \Gamma_{l}\right)}{\left(d_{0}\left(\Gamma_{l}\right) / 2\right)^{2}}<1
$$

and, thus, the estimate $(20)$ is true.

It is easy to write some simple but useful relations between a part of the operators $H_{1}^{(l)}$. In particular, we derive such relations between $H_{1}^{(l)}$ and $H_{1}^{(-l)},(-l)=\left(-l_{1},-l_{2}, \ldots,-l_{m}\right)$ where $l_{k}, k=1,2, \ldots, m$, stand for the components of the multi-index $l=\left(l_{1}, l_{2}, \ldots, l_{m}\right)$. According to our convention, $\Gamma_{(-l)}, \Gamma_{(-l)} \subset D_{(-l)}$, is a contour which is obtained from the contour $\Gamma_{l}$ by replacing all the components $\Gamma_{k}^{l_{k}}$ with the conjugate ones $\Gamma_{k}^{\left(-l_{k}\right)}$.

LEMma 2 Let $\Gamma_{l} \subset D_{l}$ be a $K_{B}$-bounded contour for which the conditions of Theorem 1 are valid. Then for any $z \in \mathbb{C} \backslash\left(\sigma^{\prime}\left(A_{0}\right) \cup \Gamma_{l}\right)$ the following equality holds true:

$$
W_{1}\left(z, \Gamma_{l}\right)\left(H_{1}^{(l)}-z\right)=\left(H_{1}^{(-l) *}-z\right)\left[W_{1}\left(\bar{z}, \Gamma_{(-l)}\right)\right]^{*} .
$$

Therefore the spectrum of $H_{1}^{(-l) *}$ coincides with the spectrum of $H_{1}^{(l)}$.

THEOREM 4 The spectrum $\sigma\left(H_{1}^{(l)}\right)$ of the operator $H_{1}^{(l)}=A_{1}+X^{(l)}$ belongs to the closed $r_{0}(B)$-vicinity $\mathcal{O}_{r_{0}}\left(A_{1}\right)$ of the spectrum of $A_{1}, \mathcal{O}_{r_{0}}\left(A_{1}\right)=\left\{z \in \mathbb{C}: \operatorname{dist}\left\{z, \sigma\left(A_{1}\right)\right\} \leq r_{0}(B)\right\}$. If a contour $\Gamma_{l} \subset D_{l}$ satisfies (14), then the complex spectrum of $H_{1}^{(l)}$ belongs to $D_{l} \cap \mathcal{O}_{r_{0}}\left(A_{1}\right)$ while outside $D_{l}$ the spectrum of $H_{1}^{(l)}$ is pure real. Moreover, the spectrum $\sigma\left(H_{1}^{(l)}\right)$ coincides with a (subset of the) spectrum of the transfer function $M_{1}\left(z, \Gamma_{l}\right)$. More precisely, the spectrum of $M_{1}\left(z, \Gamma_{l}\right)$ in $\mathcal{O}_{d_{0} / 2}\left(A_{1}\right)=\left\{z: z \in \mathbb{C}\right.$, $\left.\operatorname{dist}\left\{z, \sigma\left(A_{1}\right)\right\} \leq d_{0}\left(\Gamma_{l}\right) / 2\right\}$ is represented only by the spectrum of $H_{1}^{(l)}$, i. e. $\sigma\left(M_{1}\left(\cdot, \Gamma_{l}\right)\right) \cap \mathcal{O}_{d_{0} / 2}\left(A_{1}\right)=\sigma\left(H_{1}^{(l)}\right)$. In fact such a statement is true separately for point and continuous spectra.

THEOREM 5 Suppose that two different domains $D_{l^{\prime}}$ and $D_{l^{\prime \prime}}$ include the same subdomain $D_{k}^{l_{k}}$ for some $k=1,2, \ldots, m$, i. e., $l_{k}^{\prime}=l_{k}^{\prime \prime}=l_{k}$. Then the spectra of the operators $H_{1}^{\left(l^{\prime}\right)}$ and $H_{1}^{\left(l^{\prime \prime}\right)}$ in $D_{k}^{l_{k}}$ coincide.

Let $\Omega^{(l)}=\int_{\sigma^{\prime}\left(A_{0}\right) \cup \Gamma_{l}}\left(H_{1}^{(-l) *}-\mu\right)^{-1} K_{B}(d \mu)\left(H_{1}^{(l)}-\mu\right)^{-1}$, where as previously where $\Gamma_{l}$ stands for a $K_{B}$-bounded contour satisfying the condition (14). The operator $\Omega^{(l)}$ does not depend (for a fixed $l$ ) on the choice of such a $\Gamma_{l}$. At the same time $\Omega^{(-l)}=\Omega^{(l) *}$. The norm $\Omega^{(l)}$ satisfies the estimate

$$
\left\|\Omega^{(l)}\right\|<\frac{\mathcal{V}_{0}\left(B, \Gamma_{l}\right)}{\left(d_{0}\left(\Gamma_{l}\right) / 2\right)^{2}}<1
$$


THEOREM 6 The operators $\Omega^{(l)}$ possess the following properties(cf. [15], [20], [21]):

$$
\begin{aligned}
-\frac{1}{2 \pi \mathrm{i}} \int_{\gamma} d z\left[M_{1}\left(z, \Gamma_{l}\right)\right]^{-1} & =\left(I_{1}+\Omega^{(l)}\right)^{-1} \\
-\frac{1}{2 \pi \mathrm{i}} \int_{\gamma} d z z\left[M_{1}\left(z, \Gamma_{l}\right)\right]^{-1} & =\left(I_{1}+\Omega^{(l)}\right)^{-1} H_{1}^{(-l) *}=H_{1}^{(l)}\left(I_{1}+\Omega^{(l)}\right)^{-1}
\end{aligned}
$$

where $\gamma$ stands for an arbitrary rectifiable closed (including the points at infinity if the entry $A_{1}$ is unbounded) contour going in the positive direction around the spectrum of $H_{1}^{(l)}$ inside the set $\mathcal{O}_{d_{0}(\Gamma) / 2}\left(A_{1}\right)$. The integration over $\gamma$ is understood in the strong sense.

\section{$5 \quad$ Properties of real eigenvalues}

If $\lambda$ is a real isolated eigenvalue of the operator $H_{1}^{\left(l^{\prime}\right)}=A_{1}+X^{\left(l^{\prime}\right)}, l^{\prime}=\left(l_{1}^{\prime}, l_{2}^{\prime}, \ldots, l_{m}^{\prime}\right)$, then it is such an eigenvalue also for the remaining $2^{m-1}$ operators $H_{1}^{(l)}=A_{1}+X^{(l)}$ where $l=\left(l_{1}, l_{2}, \ldots, l_{m}\right)$ with arbitrary $l_{k}= \pm 1, k=1,2, \ldots, m$. The resolvents of every of the $2^{m}$ operators $H_{1}^{(l)}$ at $z=\lambda$ have a first order pole. Simultaneously such an eigenvalue $\lambda$ belongs to the point spectrum of the total operator $\mathbf{H}$.

An isolated real eigenvalue $\lambda$ of the operator $H_{1}^{(l)}$ can not belong to the spectrum $\sigma^{\prime}\left(A_{0}\right)$ of the entry $A_{0}$ lying outside $\bigcup_{k=1}^{m} \Delta_{k}^{0}$. Indeed, according to Theorem $₫$, the spectrum of $H_{1}^{(l)}$ for arbitrary $l$ is situated in the $r_{0}(B)$-vicinity $\mathcal{O}_{r_{0}}\left(A_{1}\right)$ of the set $\sigma\left(A_{1}\right)$ and in any case $r_{0}(B)<\frac{1}{2} \operatorname{dist}\left\{\sigma^{\prime}\left(A_{0}\right), \sigma\left(A_{1}\right)\right\}$ so that automatically $\sigma^{\prime}\left(A_{0}\right) \cap \sigma\left(H_{1}^{(l)}\right)=\emptyset$. Hence, such a $\lambda$ belongs either to the resolvent set $\rho\left(A_{0}\right)$ of the entry $A_{0}$ or it is embedded into the continuous spectrum of $A_{0}$ in $\bigcup_{k=1}^{m} \Delta_{k}^{0}$.

LEMMA 3 If a vector $\psi^{(1)} \in \mathcal{D}\left(A_{1}\right)$ is an eigenvector of $H_{1}^{(l)}$ corresponding to a real eigenvalue $\lambda \in \rho\left(A_{0}\right)$ then the vector $\Psi=\left(\psi^{(0)}, \psi^{(1)}\right) \in \mathcal{H}$ with

$$
\psi^{(0)}=-R_{0}(\lambda) B_{01} \psi^{(1)}
$$

is an eigenvector of $\mathbf{H}, \mathbf{H} \Psi=\lambda \Psi$. The converse statement is also true: if $\lambda, \lambda \in \rho\left(A_{0}\right)$, is a real eigenvalue of $H_{1}^{(l)}$ and $\mathbf{H} \Psi=\lambda \Psi$ for some $\Psi=\left(\psi^{(0)}, \psi^{(1)}\right)$ with $\psi^{(0)} \in \mathcal{D}\left(A_{0}\right)$ and $\psi^{(1)} \in \mathcal{D}\left(A_{1}\right)$, then $\psi^{(0)}$ is related to $\psi^{(1)}$ as in $(25)$ and $H_{1}^{(l)} \psi^{(1)}=\lambda \psi^{(1)}$.

If an eigenvalue $\lambda$ of $H_{1}^{(l)}$ belongs to $\Delta_{k}^{0}=\left(\mu_{k}^{(1)}, \mu_{k}^{(2)}\right)$ for some $k=1,2, \ldots, m$, then $\left|\lambda-\mu_{k}^{(i)}\right| \geq \operatorname{dist}\left\{\mu_{k}^{(i)}, \sigma\left(A_{1}\right)\right\}-r_{0}(B), \quad i=1,2$. Recall that according to our assumption the entry $A_{0}$ has no point spectrum inside $\Delta_{k}^{0}$. Since $\Delta_{k}^{0}$ is a part of the continuous spectrum of $A_{0}$, the resolvent $R_{0}(z)=\left(A_{0}-z\right)^{-1}$ for $z=\lambda \pm \mathrm{i} 0$ exists being however an unbounded operator. Nevertheless a statement analogous to Lemma 3 is valid in this case, too.

LEMMA 4 If a vector $\psi^{(1)} \in \mathcal{D}\left(A_{1}\right)$ is an eigenvector of $H_{1}^{(l)}$ corresponding to a real eigenvalue $\lambda \in \Delta_{k}^{0}=\left(\mu_{k}^{(1)}, \mu_{k}^{(2)}\right), k=1,2, \ldots, m, H_{1}^{(l)} \psi^{(1)}=\lambda \psi^{(1)}$, then either

a) $E^{0}(\mu) B_{01} \psi^{(1)}=0$ for all $\mu \leq \mu_{k}^{(2)}$

or 
b) $E^{0}(\mu) B_{01} \psi^{(1)} \neq 0$ for any $\mu \in \Delta_{k}^{0}$,

and
c) the function $\left\|E^{0}(\mu) B_{01} \psi^{(1)}\right\|$ is differentiable in $\mu$ on $\Delta_{k}^{0}$

d) $\left.\frac{d}{d \mu}\left\|E^{0}(\mu) B_{01} \psi^{(1)}\right\|\right|_{\mu=\lambda}=0$.

In both cases the vector $\psi^{(0)}$ given by (25) exists in $\mathcal{D}\left(A_{0}\right)$ and $\Psi=\left(\psi^{(0)}, \psi^{(1)}\right)$ is an eigenvector of $\mathbf{H}, \mathbf{H} \Psi=\lambda \Psi$.

The converse statement is also true. Namely, if a $\Psi=\left(\psi^{(0)}, \psi^{(1)}\right)$ with $\psi^{(0)} \in \mathcal{D}\left(A_{0}\right)$ and $\psi^{(1)} \in \mathcal{D}\left(A_{1}\right)$ is an eigenvector of $\mathbf{H}, \mathbf{H} \Psi=\lambda \Psi$, corresponding to an eigenvalue $\lambda$ of $H_{1}^{(l)}, \lambda \in \Delta_{k}^{0}$, then either the condition $(a)$ is valid or the conditions $(b-d)$ are valid. In both cases the relation (25) is retained meaning, in particular, that $\psi^{(1)} \neq 0$ and $\psi^{(1)}$ is an eigenvector of $H_{1}^{(l)}$ corresponding to the eigenvalue $\lambda$.

Let $\sigma_{p r i}\left(H_{1}^{(l)}\right)$ be the set of all real isolated eigenvalues of the operator $H_{1}^{(l)}$. As we already established, this set coincides with the part $\sigma_{p r i}\left(M_{1}\left(\cdot, \Gamma_{l}\right)\right.$ of the set of the real isolated eigenvalues of the transfer function $M_{1}\left(z, \Gamma_{l}\right)$ belonging to $\mathcal{O}_{d_{0} / 2}\left(A_{1}\right)$ for any $K_{B}$-bounded contour $\Gamma_{l}$ satisfying the condition (14).

Since in the remainder of the Section we will consider different eigenvalues $\lambda \in$ $\sigma_{p r i}\left(H_{1}^{(l)}\right)$, we will use a more specific notation, $\psi_{\lambda, j}^{(1)}, j=1,2, \ldots, m_{\lambda}$, for the respective eigenvectors of the $H_{1}^{(l)}$. The notation $m_{\lambda}, m_{\lambda} \leq \infty$, stands for the multiplicity of the eigenvalue $\lambda$. Recall that every $\psi_{\lambda, j}^{(1)}$ is an eigenvector simultaneously for all the $H_{1}^{(l)}$ and $M_{1}\left(\lambda \pm \mathrm{i} 0, \Gamma_{l}\right), l=\left(l_{1}, l_{2}, \ldots, l_{m}\right)$ with $l_{k}= \pm 1, k=1,2, \ldots, m$. In the considered case the multiplicity $m_{\lambda}$ is both the geometric and algebraic multiplicity of $\lambda$. Respective eigenvectors of the total matrix $\mathbf{H}$ will be denoted by $\Psi_{\lambda, j}, \Psi_{\lambda, j}=\left(\psi_{\lambda, j}^{(0)}, \psi_{\lambda, j}^{(1)}\right)$. It will be supposed that the $\psi_{\lambda, j}^{(1)}$ are chosen in such a way that the vectors $\Psi_{\lambda, j}$ are orthonormal, $\left\langle\Psi_{\lambda, j}, \Psi_{\lambda^{\prime}, j^{\prime}}\right\rangle=\delta_{\lambda \lambda^{\prime}} \delta_{j j^{\prime}}$. Obviously, the statements of Lemmas 3 and 9 imply that the eigenvectors $\Psi_{\lambda, j}, \lambda \in \sigma_{p r i}\left(H_{1}^{(l)}\right)$, $j=1,2, \ldots, m_{\lambda}$, form an orthonormal basis in the invariant subspace of the operator $\mathbf{H}$ corresponding to the subset $\sigma_{p r i}\left(H_{1}^{(l)}\right)$ of the point spectrum $\sigma_{p}(\mathbf{H})$ of $\mathbf{H}$.

Lemma 5 Let $\mathcal{H}_{1}^{(p r i)}, \mathcal{H}_{1}^{(p r i)} \subset \mathcal{H}_{1}$, be the closed span of the eigenvectors $\psi_{\lambda, j}^{(1)}$ i of $H_{1}^{(l)}$ corresponding to the spectrum $\sigma_{\text {pri }}\left(H_{1}^{(l)}\right), \mathcal{H}_{1}^{(p r i)}=\overline{\mathrm{V}\left\{\psi_{\lambda, j}^{(1)}, \lambda \in \sigma_{\text {pri }}\left(H_{1}^{(l)}\right), j=1,2, \ldots, m_{\lambda}\right\}}$. For any $l=\left(l_{1}, l_{2}, \ldots, l_{m}\right), l_{k}= \pm 1, k=1,2, \ldots, m$, the operator $\Omega^{(l)}$ is non-negative on the subspace $\mathcal{H}_{1}^{(\text {pri })}$.

This statement implies that one can introduce a new inner product in $\mathcal{H}_{1}^{(\text {pri })},\left[u_{1}, v_{1}\right]_{\mathcal{H}_{1}^{(p r i)}}=$ $\left\langle\left(I_{1}+\Omega^{(l)}\right) u_{1}, v_{1}\right\rangle$. Then, with the help of a theorem of N. K. BARI (Theorem VI.2.1 of [18]) one can prove the following statement.

TheOREM 7 The system of vectors $\psi_{\lambda, j}^{(1)}, \lambda \in \sigma_{\text {pri }}\left(H_{1}^{(l)}\right), j=1,2, \ldots, m_{\lambda}$, forms a Riesz basis of the subspace $\mathcal{H}_{1}^{(\text {pri) }}$. 


\section{Completeness and basis properties}

We restrict ourselves to the case where the entry $A_{1}$ has pure discrete spectrum only, i. e., the resolvent $R_{1}(z)=\left(A_{1}-z\right)^{-1}$ is a compact operator in $\mathcal{H}_{1}$ for any $z \in \rho\left(A_{1}\right)$. In this case the operators $H_{1}^{(l)}$ have compact resolvents, too. This is a consequence of Theorem V.3.17 of 19] since the difference $H_{1}^{(l)}-A_{1}=X^{(l)}$ is a bounded operator (see Theorem [1). Also, the operators $X^{(l)}$ are compact.

Denote by $\mathcal{H}_{1, \lambda}^{(l)}$ the algebraic eigenspace of $H_{1}^{(l)}$ corresponding to an eigenvalue $\lambda$. Let $m_{\lambda}$ be the algebraic multiplicity, $m_{\lambda}=\operatorname{dim} \mathcal{H}_{1, \lambda}^{(l)}, m_{\lambda}<\infty$, and $\psi_{\lambda, i}^{(l)}, i=1,2, \ldots, m_{\lambda}$, be the root vectors of $H_{1}^{(l)}$ forming a basis of the subspace $\mathcal{H}_{1, \lambda}^{(l)}$. In the following we will try to give an answer on the question when the union of such bases in $\lambda$ forms a basis of the total space $\mathcal{H}_{1}$. But, in any case, we already have an assertion regarding completeness of the system

$$
\left\{\psi_{\lambda, i}^{(l)}, \lambda \in \sigma\left(H_{1}^{(l)}\right), i=1,2, \ldots, m_{\lambda}\right\} .
$$

THEOREM 8 The closure of the linear span of the system (26) coincides with $\mathcal{H}_{1}$.

This assertion is a particular case of Theorem V.10.1 from [18].

We shall consider the case where the intersection $\left(\bigcup_{k=1}^{m} \Delta_{k}^{0}\right) \cap \sigma\left(A_{1}\right)$ includes infinitely many points and the entry $A_{1}$ is semibounded from below. This assumption means that at least the interval $\Delta_{m}^{0}$ is infinite, $\Delta_{m}^{0}=\left(\mu_{m}^{(1)},+\infty\right)$. The eigenvalues $\lambda_{i}^{\left(A_{1}\right)}, i=1,2, \ldots$, of the operator $A_{1}$ will be enumerated in increasing order, $\lambda_{1}^{\left(A_{1}\right)}<\ldots<\lambda_{i}^{\left(A_{1}\right)}<\lambda_{i+1}^{\left(A_{1}\right)}<\ldots$; $\lim _{i \rightarrow \infty} \lambda_{i}^{\left(A_{1}\right)}=+\infty$ exists.

Suppose further that there is a number $i_{0}$ such that for any $i \geq i_{0}$ and for some fixed $r>r_{0}(B)$

$$
\lambda_{i}^{\left(A_{1}\right)}-\lambda_{i-1}^{\left(A_{1}\right)}>2 r
$$

Let $\gamma_{0}$ be a circle centered at $z=\left(\lambda_{1}^{\left(A_{1}\right)}+\lambda_{i_{0}-1}^{\left(A_{1}\right)}\right) / 2$ and having the radius $\left(\lambda_{i_{0}-1}^{\left(A_{1}\right)}-\lambda_{1}^{\left(A_{1}\right)}\right) / 2+r$ while the $\gamma_{i}$ for $i \geq i_{0}$ are the circles with centers $\lambda_{i}^{\left(A_{1}\right)}$ and the radius $r$. Let us introduce the projections $\mathrm{Q}_{i}^{(l)}=-\frac{1}{2 \pi \mathrm{i}} \int_{\gamma_{i}} d z\left(H_{1}^{(l)}-z\right)^{-1}, i=0, i_{0}, i_{0}+1, \ldots$ Every projection $\mathrm{Q}_{i}^{(l)}$ represents a sum of the eigenprojections corresponding to the eigenvalues $\lambda^{(l)}$ of $H_{1}^{(l)}$ lying inside $\gamma_{i}$ and $\mathrm{Q}_{i}^{(l)} \mathrm{Q}_{j}^{(l)}=\delta_{i j} \mathrm{Q}_{i}^{(l)}$. The subspaces $\mathcal{N}_{i}^{(l)}=\mathrm{Q}_{i}^{(l)} \mathcal{H}_{1}$ are invariant under $H_{1}^{(l)} ; \operatorname{dim} \mathcal{N}_{i}^{(l)}$ coincides with a sum of algebraic multiplicities of the eigenvalues $\lambda^{(l)}$ lying inside $\gamma_{i}$. We introduce also the (orthogonal) projections $\mathrm{P}_{i}^{\left(A_{1}\right)}=-\frac{1}{2 \pi \mathrm{i}} \int_{\gamma_{i}} d z\left(A_{1}-z\right)^{-1}, i=0, i_{0}, i_{0}+1, \ldots$.

LEMMA 6 Under the condition (27) the sequence $\mathcal{N}_{i}^{(l)}, i=0, i_{0}, i_{0}+1, \ldots$, is $\omega$-linearly independent and complete in $\mathcal{H}_{1}$. If instead of (27) the condition

$$
\lambda_{i}^{\left(A_{1}\right)}-\lambda_{i-1}^{\left(A_{1}\right)}>2 r>4 r_{0}(B) \quad \forall i \geq i_{0},
$$

is satisfied then $\operatorname{dim} \mathcal{N}_{i}^{(l)}=\operatorname{dim} \mathrm{P}_{i}^{\left(A_{1}\right)} \mathcal{H}_{1}, i=0, i_{0}, i_{0}+1, \ldots$ 
THEOREM 9 Assume $\lambda_{i+1}^{\left(A_{1}\right)}-\lambda_{i}^{\left(A_{1}\right)} \rightarrow \infty$ as $i \rightarrow \infty$. Let $i_{0}$ be a number starting from which the inequality (28) holds. Then the following limit exists

$$
s-\lim _{n \rightarrow \infty} \sum_{i=0, i \geq i_{0}}^{n} \mathrm{Q}_{i}^{(l)}=I_{1} .
$$

Additionally, assume that

$$
\sum_{i=1}^{\infty}\left(\lambda_{i+1}^{\left(A_{1}\right)}-\lambda_{i}^{\left(A_{1}\right)}\right)^{-2}<\infty .
$$

Then $(29)$ is true for any renumbering of $\mathrm{Q}_{i}^{(l)}$. Moreover, there exists a constant $C$ such that $\left\|\sum_{i \in \mathcal{I}} \mathrm{Q}_{i}^{(l)}\right\| \leq C$ for any finite set $\mathcal{I}$ of integers $i=0, i \geq i_{0}$.

This theorem represents a slightly extended statement of Theorems V.4.15 and V.4.16 of [19].

REMARK 1 Eq. (29) implies that

$$
s-\lim _{n \rightarrow \infty} \sum_{i=0, i \geq i_{0}}^{n} \sum_{\lambda \in \operatorname{Int} \gamma_{i}} \mathrm{P}_{\lambda}^{(l)}=I_{1}
$$

where $\lambda$ stand for the eigenvalues of the operator $H_{1}^{(l)}$ and $\mathrm{P}_{\lambda}^{(l)}$ for the respective eigenprojections. If, additionally, the inequality (30) holds and all the eigenvalues $\lambda_{i}^{\left(A_{1}\right)}$ are simple, then one can renumber the eigenprojections $\mathrm{P}_{\lambda}^{(l)}$ in Eq. (31) in any way (see Theorem V.4.16 of [19]).

THEOREM 10 As before, assume $\Delta_{m}=\left(\mu_{m}^{(1)},+\infty\right)$. Also, suppose that there is a $K_{B^{-}}$ bounded contour $\Gamma_{l} \subset D_{l}$ satisfying (14) and such that a part of its component $\Gamma_{m}^{l_{m}}$ coincides with the ray $\tilde{\Delta}_{m}^{0}=\left[\mu_{0}, \mathrm{i} b_{0}+\infty\right)$ where $\mu_{0} \in D_{m}^{l_{m}}, \mu_{0}=a_{0}+\mathrm{i} b_{0}$ with $a_{0}, b_{0} \in \mathbb{R}$. Additionally, suppose that the remaining part $\tilde{\Gamma}_{l}=\Gamma_{l} \backslash \tilde{\Delta}_{m}^{0}$ of the contour $\Gamma_{l}$ belongs to the half-plane $\operatorname{Re} \mu<a_{0}$, and for $\mu \in \tilde{\Delta}_{m}^{0}\left\|K_{B}^{\prime}(\mu)\right\| \leq \tilde{C}(1+|\operatorname{Re} \mu|)^{-\theta}$, with $\tilde{C}>0$ and $\theta>1$. Also, let the condition (30) be valid. The sequence of the subspaces $\mathcal{N}_{i}^{(l)}=\mathrm{Q}_{i}^{(l)} \mathcal{H}_{1}, \quad i=0, i_{0}, i_{0}+1, \ldots$, forms a basis of the space $\mathcal{H}_{1}$, quadratically close to an orthogonal one. If, additionally, $\operatorname{dim} \mathrm{P}_{i}^{\left(A_{1}\right)} \mathcal{H}_{1} \leq n$, for some $n \in \mathbb{N}$, the same for all $i=0, i_{0}, i_{0}+1, \ldots$, then the union of orthonormal vector bases of the subspaces $\mathcal{N}_{i}^{(l)}, i=0, i_{0}, i_{0}+1, \ldots$, forms a Bari basis of the space $\mathcal{H}_{1}$.

\section{References}

[1] Jaffe, R. L., and Low, F. E.: Connection between Quark-Model Eigenstates and LowEnergy Scattering, Phys. Rev. D19 (1979), 2105-2118. 
[2] Simonov, Yu. A.: Hadron-Hadron Interaction in the Compound-Bag Model, Yadernaya Fiz. 36 (1982), 722-731 [Russian].

[3] Okubo, S.: Diagonalization of Hamiltonian and Tamm-Dancoff equation, Progr. Theor. Phys. 12 (1954), 603-622.

[4] Glöckle, W., and Müller, L.: Relativistic Theory of Interacting Particles, Phys. Rev. C 23 (1981), 1183-1195.

[5] Korchin, A. Yu., and Shebeko, A. V.: The Method of Okubo's Effective Operators and Relativistic Model of Nuclear Structure, Phys. At. Nucl. 56 (1993), 1663-1671.

[6] Malyshev, V. A., and Minlos, R. A.: Invariant Subspaces of Clustering Operators. I., J. Stat. Phys. 21 (1979), 231-242; Invariant Subspaces of Clustering Operators. II., Comm. Math. Phys. 82 (1981), 211-226.

[7] Pavlov, B. S., and Shushkov, A. A.: The Theory of Extensions, and Null-Range Potentials with Internal Structure, Math. USSR Sb. 65 (1990), 147-184.

[8] McKellar, B. H. J., and McKay C. M.: Formal Scattering Theory for Energy-Dependent Potentials, Aust. J. Phys. 36 (1983), 607-616.

[9] Schmid, E. W.: The Problem of Using Energy-Dependent Nucleon-Nucleon Potentials in Nuclear Physics, Helv. Phys. Acta 60 (1987), 394-397.

[10] Motovilov, A. K.: Potentials Appearing after the Removal of an Energy-Dependence and Scattering by Them, In: Proc. of the Intern. Workshop "Mathematical aspects of the scattering theory and applications", St. Petersburg University, St. Petersburg, 1991. P. 101-108.

[11] Motovilov, A. K.: Removal of the Resolvent-like Energy Dependence from Interactions and Invariant Subspaces of a Total Hamiltonian, J. Math. Phys. 36 (1995), 6647-6664 (LANL E-print funct-an/9606002); Elimination of Energy from Interactions Depending on It as a Resolvent, Theor. Math. Phys. 104 (1995), 989-1007 (LANL E-print nucl-th/9505030).

[12] Braun, M. A.: On Relation between Quasipotential Equation and Schrödinger Equation, Teor. Mat. Fiz. 72 (1987), 394-402 [Russian].

[13] Adamjan, V. M., and Langer, H.: Spectral Properties of a Class of Operator-Valued Functions, J. Operator Theory 33 (1995), 259-277.

[14] Adamyan, V., Langer, H., Mennicken, R., and Saurer, J.: Spectral Components of Selfadjoint Block Operator Matrices with Unbounded Entries, Math. Nachr. 178 (1996), 43-80.

[15] Mennicken, R., and Shkalikov, A. A.: Spectral Decomposition of Symmetric Operator Matrices, Math. Nachr. 179 (1996), 259-273.

[16] Reed, M., and Simon, B.: Methods of Modern Mathematical Physics, III: Scattering theory, Academic Press, N.Y., 1979.

[17] Motovilov, A. K.: Representations for the Three-Body T-Matrix, Scattering Matrices and Resolvent on Unphysical Energy Sheets, Math. Nachr. 187 (1997), 147-210 (LANL E-print funct-an/9509003). 
[18] Gohberg, I. C., and Krein, M. G.: Introduction to the Theory of Linear Non-selfadjoint Operators, American Mathematical Society, Providence, 1988.

[19] Kato, T. Perturbation Theory for Linear Operators, New York: Springer-Verlag, 1966.

[20] Virozub, A. I., and Matsaev, V. I.: The Spectral Properties of a Certain Class of Selfadjoint Operator Functions, Funct. Anal. Appl. 8 (1974), 1-9.

[21] Markus, A. S., and Matsaev, V. I.: On the Basis Property for a Certain Part of the Eigenvectors and Associated Vectors of a Selfadjoint Operator Pencil, Math. USSR Sb. 61 (1988), 289-307.

AMS Classification Numbers: Primary 47A56, 47Nxx; Secondary 47N50, 47A40. 